\title{
SPL Blood Form Product Type Terminology
}

National Cancer Institute

\section{Source}

National Cancer Institute. SPL Blood Form Product Type Terminology. NCI Thesaurus.

Code C133243.

Terminology used to qualify the information pertaining to blood form product types in the framework of the Structured Product Labeling documents. 